\title{
Electron emission of cathode holder of vacuum diode of an intense electron-beam accelerator and its effect on the output voltage
}

\author{
Xin-Bing Cheng, Jin-Liang Liu, Hong-Bo Zhang, Zhi-Qiang Hong, and Bao-Liang Qian \\ College of Opto-electronic Science and Engineering, National University of Defense Technology, \\ Changsha, Hunan, 410073, People's Republic of China \\ (Received 19 December 2010; published 28 April 2011)
}

\begin{abstract}
The vacuum diode which is used to generate relativistic electron beams is one of the most important parts of a pulsed-power modulator. In this paper, the electron emission of cathode holder of a vacuum diode and its effect on the output voltage is investigated by experiments on an intense electron-beam accelerator with $180 \mathrm{~ns}$ full width at half maximum and 200-500 kV output voltage. First, the field emission is analyzed and the electric field of the vacuum chamber is calculated. Then, the flatness of the output voltage is discussed before and after adding an insulation plate when a water load is used. It is found that the electron emission at the edges of the cathode holder is the main reason to cause the change of the flatness. Last, a piece of polyester film is used as a target to further show the electron emission of the cathode holder. This analysis shows that decreasing the electron emission of the cathode holder in such a pulse power modulator could be a good way to improve the quality of the output voltage.
\end{abstract}

DOI: 10.1103/PhysRevSTAB.14.040402

PACS numbers: $84.30 . \mathrm{Ng}, 84.70 .+\mathrm{p}, 87.56 . \mathrm{bd}$

\section{INTRODUCTION}

Pulsed-power technology has been used in many fields, such as high-power microwave (HPM) generation [1-4], $Z$ pinch [5-8], electron-beam pumped high-power laser $[9,10]$, ion implantation for material modifications [11], and so on. At present, as the development of the pulsed power, the full width at half maximum (FWHM) of the output pulse of many pulsed-power facilities exceeds $100 \mathrm{~ns}$, output voltage is several hundred kilovolts, even megavolt, and output current is up to hundred kiloamperes. For example, as the development of the narrow band highpower microwave (HPM) device, efforts have been made on increasing the output power $(>1 \mathrm{GW})$, HPM pulse duration $(>100 \mathrm{~ns})$, and conversion efficiency of these devices [12]. Also, the typical pulse power facility for the electron-beam pumped high-power laser is Naval Research Laboratory's Electra generator with $140 \mathrm{~ns}$ FWHM, $500 \mathrm{kV}$ voltage, and $110 \mathrm{kA}$ current [9], which is used to pump $\mathrm{He}-\mathrm{Ar}, \mathrm{Ar}-\mathrm{Xe}, \mathrm{XeCl}, \mathrm{XeF}, \mathrm{ArF}$, and $\mathrm{KrF}$ lasers [13]. In these applications, the vacuum diodes are among the most important parts of the pulsed-power modulator. It converts several hundred kilovolt and nanosecond voltage pulse into relativistic electron beams to drive a high-power microwave source [1-4], or to generate pulsed lasers [13]. To improve the quality of the electron beams, the output voltage of these pulse power facilities is very important, because the quality of the electron beams is directly

Published by the American Physical Society under the terms of the Creative Commons Attribution 3.0 License. Further distribution of this work must maintain attribution to the author(s) and the published article's title, journal citation, and DOI. decided by the flatness of the output voltage. In our previous paper [14], the effect of the transition section of an intense electron-beam accelerator (IEBA) on the output voltage has been discussed. According to our recent experiments, it is found that the electron emission of the cathode holder also has a great effect on the output characteristic of the IEBA.

To clearly show the electron emission of the cathode holder of the vacuum diode, a vacuum diode with adjustable cathode-anode gap is not a good choice, because the emission current density of the diode is so strong that the electron emission of the cathode holder could be ignored, so a dummy load is needed. Also, to characterize these kinds of IEBA accurately, one needs a matched dummy load that has sufficiently broad bandwidth and that can withstand high voltage (several hundred kilovolts) and can handle large current (tens of kiloamperes) [15]. The metal thread wound around a cylinder to achieve certain conductivity is usually chosen as the dummy load. However, the metal thread load has a larger inductance $(100 \mathrm{nH})$, and it could be destroyed by the field enhancement. So the life of the metal thread load is short. Now, a water load with low inductance, high power, and constant resistance is usually used, and the water is a kind of aqueous resistive solution of copper sulfate (CuSO4) or sodium chloride $(\mathrm{NaCl})$ of a concentration that is adjusted to match the impedance of the generator $[15,16]$.

In this paper, the electron emission phenomenon of the cathode holder of a vacuum diode under hundreds of kilovolt for a IEBA is investigated by using an axial current water load, and its effect on the output voltage is also discussed, especially the flatness of the flattop of the output voltage. By varying the gas pressure of the main switch, distance between the cathode holder and water load, the 
factors which affect the flatness are discussed. In Sec. II', we describe the pulsed-power modulator, especially its vacuum chamber. The field emission analysis and electric field calculation are present in Sec. III. Then, the output voltage of the experiments, flatness analysis of the output voltage, and photograph of the electron bombardment are given in Sec. IV. Finally, the conclusion is given in Sec. V.

\section{EXPERIMENTAL SETUP}

The experiment is performed on an IEBA which is designed by our laboratory [17], and it is capable of generating a quasi-square wave with voltage of $200-500 \mathrm{kV}$ by adjusting the gas pressure of the main switch, and FWHM of $180 \mathrm{~ns}$ when connected to impedance matched load. This IEBA consists of a $50 \mathrm{kV}, 12 \mathrm{uF}$ primary storage capacitor, a field distortion switch, a spiral strip type pulsed transformer, main switch with sulfur hexafluoride $\left(\mathrm{SF}_{6}\right)$ as insulating medium, spiral-type Blumlein pulse forming line (BPFL), vacuum diodes chamber, mechanical and molecular pumps, and voltage and current diagnostic system.

In order to investigate the electron emission of the cathode holder and to characterize the IEBA, a high-power water load is used to instead of the electron-beam diode, and it consists of electrodes, insulating support, and aqueous resistive solution. Also, to clearly show the electron emission, an insulation plate is added between the cathode holder and the water load during the experiments. Figure 1 shows the vacuum chamber cross section of the IEBA and Fig. 2 shows the photograph of the vacuum chamber. The cathode holder which is made of stainless steel is discoid. Before the experiments, the load resistance is adjusted to match the impedance of BPFL, the water loads are placed between the cathode holder and the outer cylinder of the BPFL which is grounded, and then pumping down the vacuum chamber to a pressure of $10^{-4}$ Torr by means of combination of mechanical and molecular pumps. The gas pressures of the main switch are varied within the range of 0.20-0.45 MPa. The output voltage on the load is diagnosed by the resistive divider shown in Fig. 1.

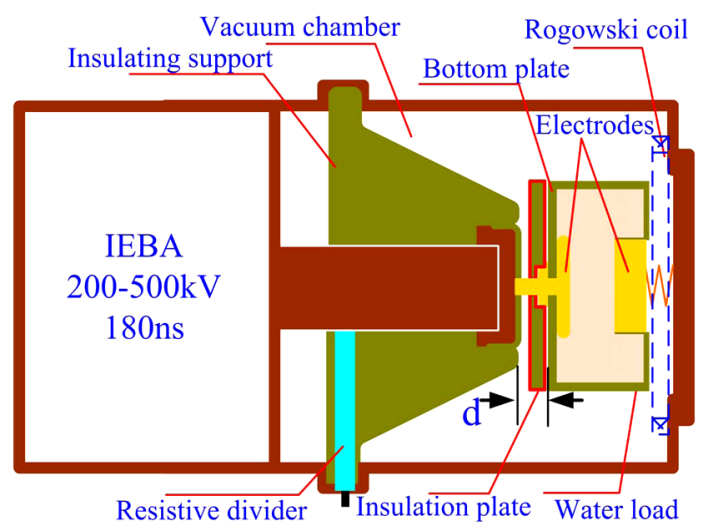

FIG. 1. Schematic of the cross section of the vacuum chamber.

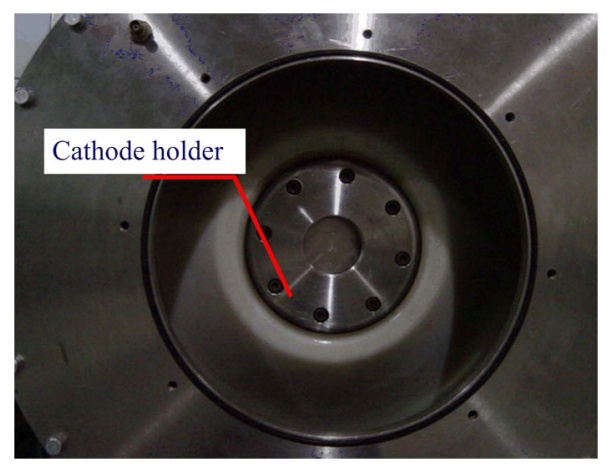

FIG. 2. Photograph of the vacuum chamber.

\section{THEORETICAL ANALYSIS}

In the vacuum chamber of the IEBA, the vacuum is pumped down a pressure of $10^{-4}$ Torr, and the electrons are emitted by field enhancement. It is known that the field emission can be described by the well-known FowlerNordheim (FN) model [18],

$$
J=\frac{1.56 \times 10^{-6} E^{2}}{\phi} \exp \left(\frac{-6.83 \times 10^{7} \phi 3 / 2}{E}\right)
$$

where $J$ is the field emission current density $\left(\mathrm{A} / \mathrm{cm}^{2}\right)$, $\phi$ is the work function of emitters $(\mathrm{eV})$, and $E$ is the microscopic local electric field at the emission sites $(\mathrm{V} / \mathrm{cm})$. The actual value of $E$ could not be measured directly, and it is closely related to the applied voltage $U$ by setting

$$
E=\beta f(U),
$$

where $\beta$ is the field enhancement factor, according to the FN model, the field mission property is sensitive to the work function $\phi$ and the electric field. Here, for stainless steel, the work function is about $4.4 \mathrm{eV}$ [19].

To obtain the electric field, the finite element method is used. Here, the voltage of the cathode holder is assumed as 200, 250, 300, 350, 400, and $450 \mathrm{kV}$, respectively. Figure 3 shows the typical electric potential distribution for $300 \mathrm{kV}$ before adding the insulation plate and after adding the insulation plate. Figure 4 shows the maximum electric field which is at the edges of the cathode holder as a function of voltage before and after adding the insulation plate.

In Fig. 4, after adding the insulation plate, the electric field is much bigger, and the relationship between the maximum electric field and the voltage can be obtained by linear fit of the curve:

$$
\begin{aligned}
& E_{M_{B}}=f(U)=0.228 U \\
& E_{M_{A}}=f(U)=0.332 U,
\end{aligned}
$$

where $E_{M_{B}}$ and $E_{M_{A}}$ are the maximum electric field before and after adding the insulation plate, respectively, so for 

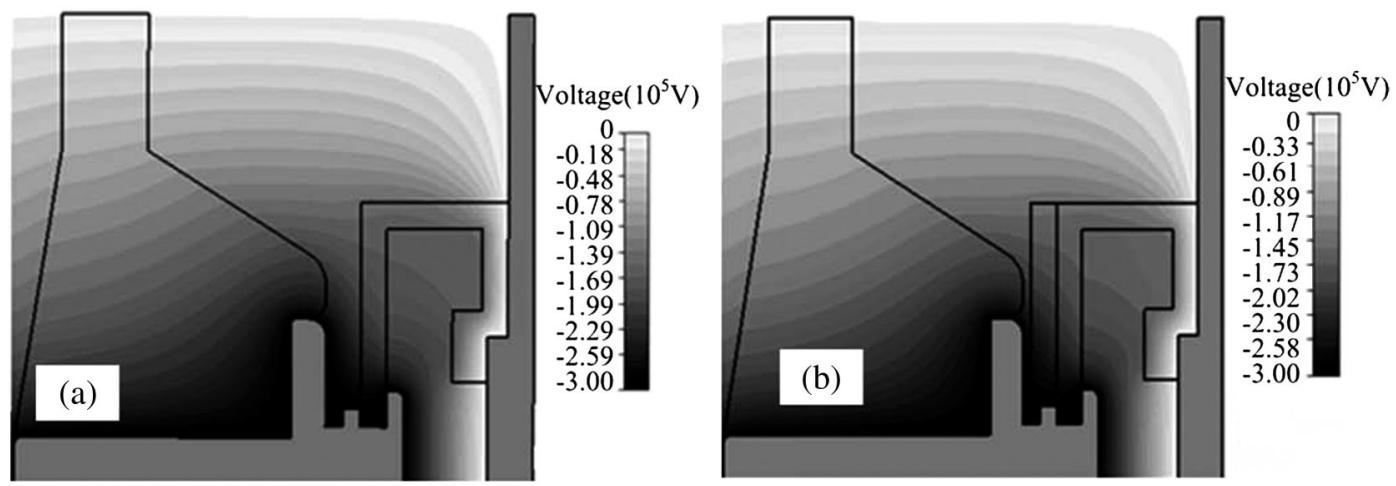

FIG. 3. The typical electric potential distribution for $300 \mathrm{kV}$ (a) before adding the insulation plate and (b) after adding the insulation plate.

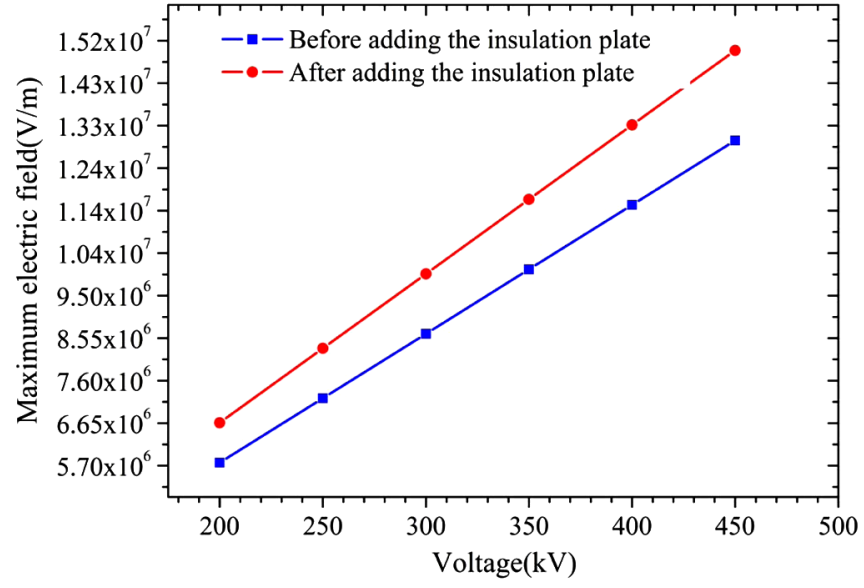

FIG. 4. Dependence of maximum electric field on the voltage before and after adding the insulation plate.

the constant cathode holder, field enhancement factor $(\beta)$ and the adding voltage are dominant factors affecting the emission property. Here, the field enhancement factor is the same, for a constant voltage, the field emission current density after adding the insulation plate is much larger than that before adding the insulation plate.

\section{RESULTS AND DISCUSSION}

\section{A. Experiments results}

First, the water load is placed in the vacuum chamber, and no insulation plate is added between the cathode holder and bottom plate of the water load. So the distance $d$ is $20 \mathrm{~mm}$ in Fig. 1. In Fig. 5 are shown the output voltage waveforms of the IEBA when the gas pressure $\left(P_{M}\right)$ of the main switch are $0.20,0.25,0.30,0.35,0.40,0.45 \mathrm{MPa}$, respectively. Here the load resistances are all $15 \Omega$. To quantify the waveform of the output voltage and to describe the fluctuation of the flattop of the wave, first peaking voltage $\left(V_{1}\right)$, second peaking voltage $\left(V_{2}\right)$, and peaking voltage $\left(V_{P}\right)$ are used (Fig. 5). Results of these voltages

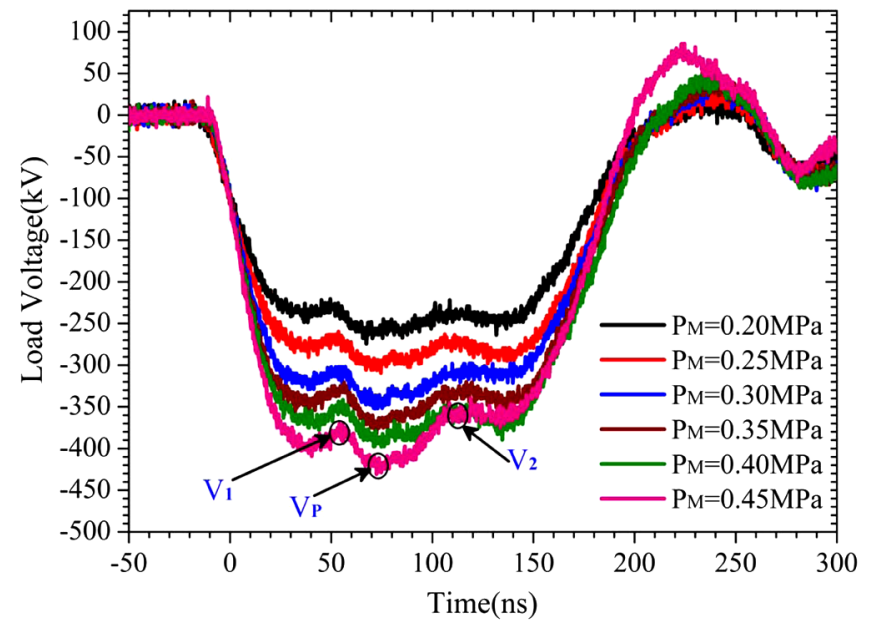

FIG. 5. Output voltage of the IEBA at different gas pressure of main switch before adding the insulation plate $(d=20 \mathrm{~mm})$.

before adding the insulation plate with different gas pressure are shown in Table I. From Fig. 5 and Table I, one can see that the output voltage is increased with the increment of the gas pressure, and the output voltage wave is a quasisquare wave with FWHM of about $180 \mathrm{~ns}$ and rise time of $30 \mathrm{~ns}$ except that there is fluctuation on the flattop. For example, there is a step in front of the main pulse, which is caused by the transition section of the BPFL [14].

TABLE I. Results of the experiments before adding the insulation plate $(d=20 \mathrm{~mm})$ for different gas pressure.

\begin{tabular}{lccc}
\hline \hline$P_{M}(\mathrm{MPa})$ & $V_{1}(\mathrm{kV})$ & $V_{P}(\mathrm{kV})$ & $V_{2}(\mathrm{kV})$ \\
\hline 0.20 & 230.6 & 258.8 & 234.8 \\
0.25 & 267.8 & 300.0 & 272.8 \\
0.30 & 305.8 & 344.2 & 306.7 \\
0.35 & 327.4 & 370.1 & 331.2 \\
0.40 & 349.0 & 392.8 & 352.0 \\
0.45 & 381.0 & 420.0 & 358.2 \\
\hline \hline
\end{tabular}




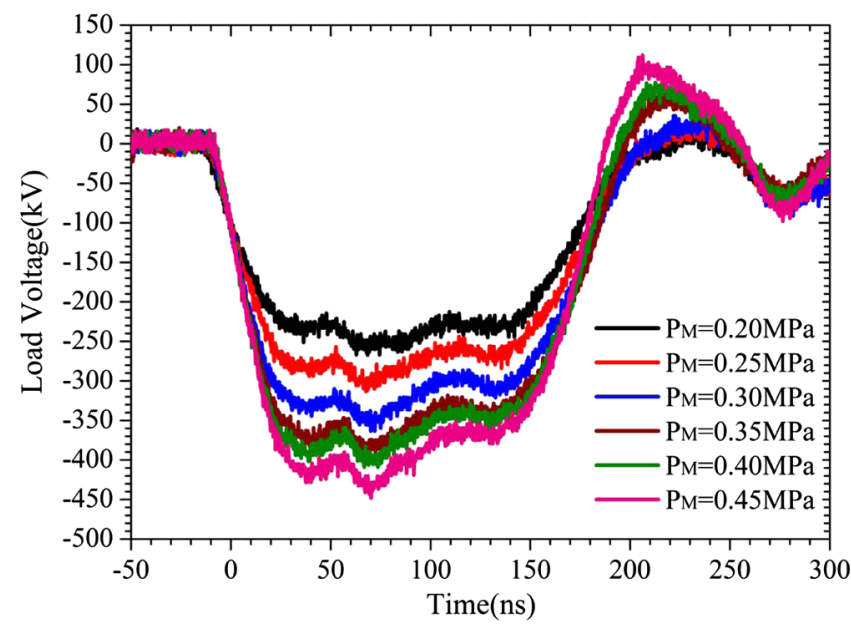

FIG. 6. Output voltage of the IEBA at different gas pressure of the main switch after adding the insulation plate $(d=5 \mathrm{~mm})$.

However, if $P_{M}$ is smaller than $0.40 \mathrm{MPa}$, the first peaking voltages are all almost equal to second peaking voltage. For example, $V_{1}$ is $305.8 \mathrm{kV}$ and $V_{2}$ is $306.7 \mathrm{kV}$ when $P_{M}$ is $0.30 \mathrm{MPa}$. If the gas pressure is up to $0.45 \mathrm{MPa}$, the second peaking voltage is reduced, $381 \mathrm{kV}$ for $V_{1}$ and $358 \mathrm{kV}$ for $V_{2}$.

To investigate the difference between the lower gas pressure and higher gas pressure, and to show the effect of the electron emission of cathode holder on the output voltage, an insulation plate with thickness of $15 \mathrm{~mm}$ is placed between the cathode holder and bottom plate of the water load, which is shown in Fig. 1, so the distance $(d)$ between the cathode holder and insulation plate is $5 \mathrm{~mm}$. Then, according to the same experiments above, the output voltage waveforms of IEBA after adding the insulation plate can be obtained in Fig. 6.

Here, the output voltage waveforms are also quasisquare wave with FWHM of about 180 ns and rise time of $30 \mathrm{~ns}$, which are similar as that in Fig. 5, and there is also a step in front of the main pulse. Table II shows the values of first peaking voltage $\left(V_{1}\right)$, second peaking voltage $\left(V_{2}\right)$, and peaking voltage $\left(V_{P}\right)$ at different gas pressure. Notably, the output voltage also has its own characteristics. When $P_{M}$ is larger than $0.20 \mathrm{MPa}$, there is a marked difference between the first peaking voltage $\left(V_{1}\right)$ and the second

TABLE II. Results of the experiments after adding the insulation plate $(d=5 \mathrm{~mm})$ for different gas pressure.

\begin{tabular}{lccc}
\hline \hline$P_{M}(\mathrm{MPa})$ & $V_{1}(\mathrm{kV})$ & $V_{P}(\mathrm{kV})$ & $V_{2}(\mathrm{kV})$ \\
\hline 0.20 & 220.8 & 255.0 & 222.5 \\
0.25 & 275.8 & 302.1 & 254.3 \\
0.30 & 322.2 & 350.2 & 293.8 \\
0.35 & 355.2 & 382.0 & 329.0 \\
0.40 & 368.8 & 400.3 & 340.4 \\
0.45 & 400.6 & 435.1 & 363.6 \\
\hline \hline
\end{tabular}

peaking voltage $\left(V_{2}\right)$. The higher the gas pressure, the bigger the difference. For example, if the gas pressure is up to $0.25 \mathrm{MPa}, 275.8 \mathrm{kV}$ for $V_{1}$ and $254.3 \mathrm{kV}$ for $V_{2}$, this is very different from that in Fig. 5. So, the output voltage waveform is obviously affected by adding the insulation plate.

\section{B. Discussion}

To show the differences of the experiment results between before adding the insulation plate and after adding the insulation, and to quantify the flatness of the output voltage waveform of the IEBA, the variance of the flattop of the output voltage is defined as

$$
\begin{aligned}
& \eta_{1}=\frac{\left|V_{1}-V_{2}\right|}{V_{2}} \times 100 \% \\
& \eta_{2}=\frac{\left|V_{P}-V_{2}\right|}{V_{2}} \times 100 \% \\
& \eta_{3}=\frac{\left|V_{1}-V_{P}\right|}{V_{P}} \times 100 \% .
\end{aligned}
$$

Obviously, if $\eta_{1}=\eta_{2}=\eta_{3}=0$, the output voltage waveform is a well-shaped square wave and there is no fluctuation on the flattop. Meanwhile, according to Tables I and II, and Eqs. (5)-(7), the variance can be obtained, which are all shown in Figs. 7-9, respectively.

The variance $\eta_{1}$ before adding the insulation plate $(d=20 \mathrm{~mm})$ and after adding the insulation plate $(d=5 \mathrm{~mm})$ is shown in Fig. 7. It is found that the variance after adding the insulation is much larger than that before adding the insulation plate. Before adding the insulation plate, $\eta_{1}$ is less than $2 \%$ when the first peaking voltage $\left(V_{1}\right)$ is less than $349 \mathrm{kV}$, but when $V_{1}$ is up to $381 \mathrm{kV}, \eta_{1}$ is

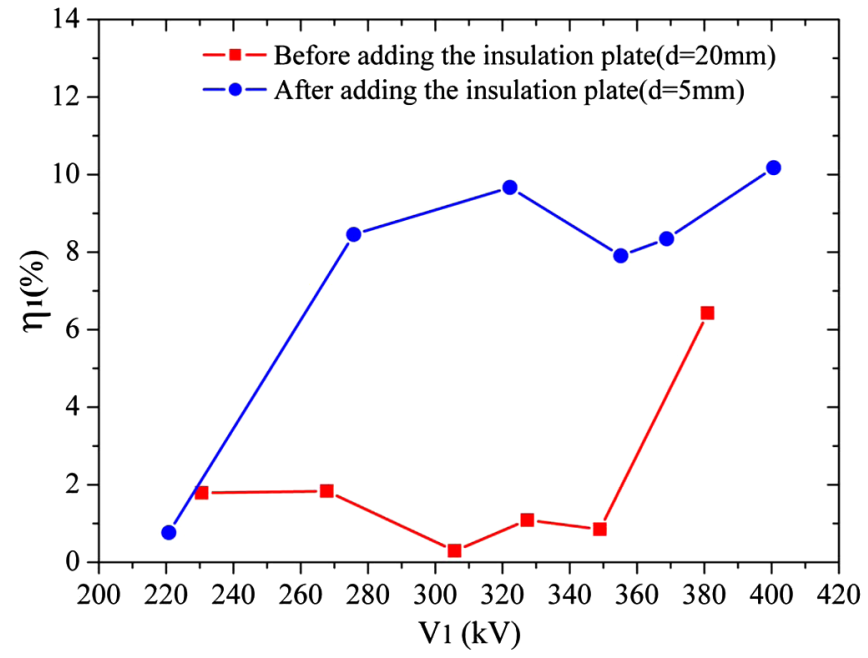

FIG. 7. Dependence of variance $\eta_{1}$ on the first peaking voltage $\left(V_{1}\right)$ before and after adding the insulation plate. 


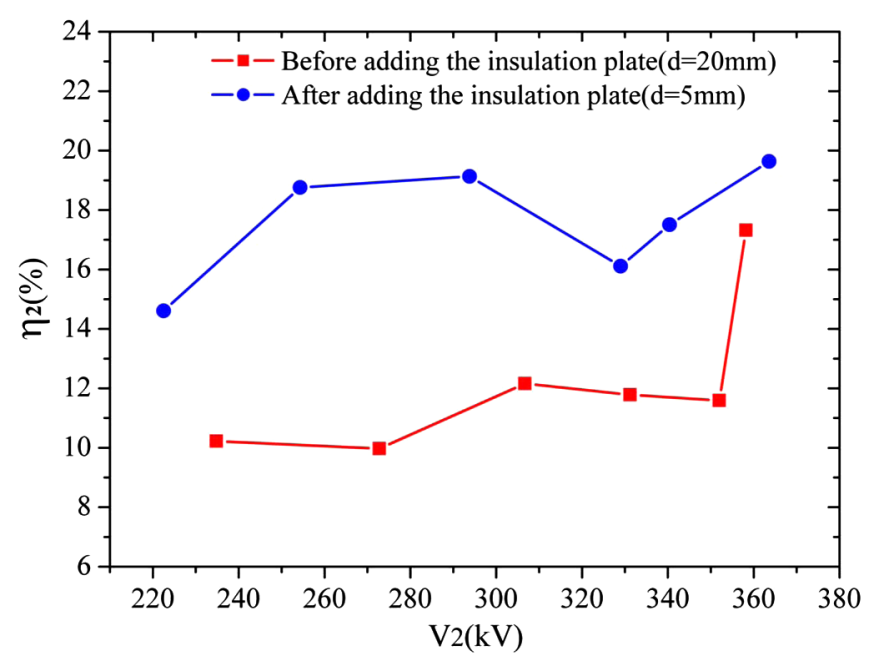

FIG. 8. Dependence of variance $\eta_{2}$ on the second peaking voltage $\left(V_{2}\right)$ before and after adding the insulation plate.

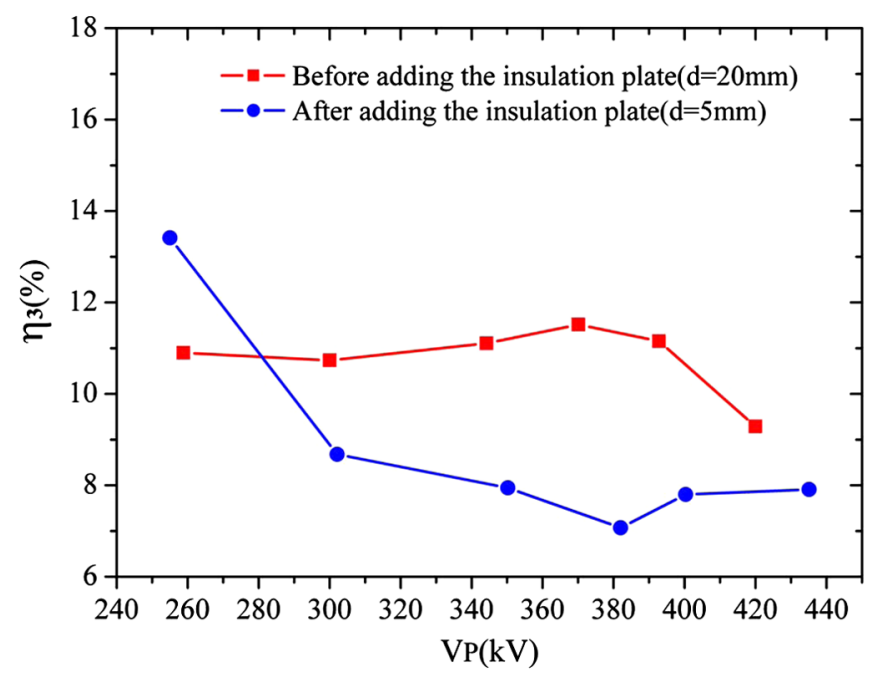

FIG. 9. Dependence of variance $\eta_{3}$ on the peaking voltage $\left(V_{P}\right)$ before and after adding the insulation plate.

quickly increased to $6.4 \%$. However, after adding the insulation plate, $\eta_{1}$ is larger than $8 \%$ when $V_{1}$ is larger than $275.8 \mathrm{kV}$. When $V_{1}$ is up to $400.6 \mathrm{kV}, \eta_{1}$ is up to $10 \%$. Notably, the insulation plate has a great effect on the flatness of the flattop of the output voltage, which increases the variance $\eta_{1}$. But the effect is insignificant at lower voltage. In other words, if distance $d$ between the cathode holder and the bottom plate of the load is long enough, the variance $\eta_{1}$ would be increased when the output voltage is up to a larger threshold value, and which value the variance is changed obviously. Contrarily, if $d$ is small enough, the threshold voltage value becomes smaller. It is $349 \mathrm{kV}$ for $d$ of $20 \mathrm{~mm}$ and $275.8 \mathrm{kV}$ for $d$ of $5 \mathrm{~mm}$.

In Fig. 8 , the variance $\eta_{2}$ before adding the insulation plate and after adding the insulation plate as a function of second peaking voltage $\left(V_{2}\right)$ is also obtained. It is shown that the variance $\eta_{2}$ is much larger than $\eta_{1}$ in Fig. 7 , before adding the insulation plate, the variance $\eta_{2}$ is about $12 \%$ when $V_{2}$ is smaller than $352 \mathrm{kV}, V_{2}$ is up to $358.2 \mathrm{kV}, \eta_{2}$ is quickly increased to $17.3 \%$, and the curve shape is similar with $\eta_{1}$ in Fig. 7. Also after adding the insulation plate, the curve shape of $\eta_{2}$ is similar to $\eta_{1}$ in Fig. 7, but the value is much larger than $\eta_{1}$. The variance $\eta_{2}$ after adding the insulation plate is $14.6 \%$ and $19.6 \%$, respectively, when $V_{2}$ is 254.3 and $363.6 \mathrm{kV}$. So, the variance $\eta_{2}$ is also increased by adding the insulation plate, and the output voltage waveform (Fig. 6) is much worse than that before adding the insulation plate (Fig. 5).

So, before adding the insulation plate, $\eta_{2}$ is larger than $\eta_{1}$, it is caused by the characteristics of the IEBA, including the transition section of BPFL, stray inductance of the load, distance between the cathode holder and water load, and other stray parameters. But most notably, the difference of variance $\eta_{1}\left(\eta_{2}\right)$ between before adding the insulation plate and after adding the insulation plate is caused by the insulation plate. In other words, the insulation plate is one of the reasons to affect the flatness of the output voltage. So it is deduced that, before adding the insulation plate, the bottom plate of the water load also affects the flatness of the output voltage of the IEBA in Fig. 1.

For variance $\eta_{3}$ in Fig. 9, it describes the relationship between the first peaking voltage and peaking voltage. In fact, the step is formed by the transition section of the BPFL [14]. So before adding the insulation plate $(d=20 \mathrm{~mm})$, variance $\eta_{3}$ is almost the same, it is about $11 \%$. However, when peaking voltage $\left(V_{P}\right)$ is increased to $420 \mathrm{kV}, \eta_{3}$ is decreased to $9 \%$. It is also proved that the output voltage is affected little when the first peaking voltage is less than $349 \mathrm{kV}$. Meanwhile, after adding the insulation plate $(d=5 \mathrm{~mm})$, the variance $\eta_{3}$ is decreased obviously, and it is about $7.9 \%$ when $V_{P}$ is larger than $302.1 \mathrm{kV}$. Also, at a lower peaking voltage $\left(V_{P}=255 \mathrm{kV}\right)$, the variance is about $13.4 \%$ which is approximate to that before adding the insulation plate. These further prove that the distance $d$ between the cathode holder and the bottom plate of the load has a great effect on the flatness of output voltage and the threshold voltage.

Through the above experiments, some basics conclusions could be obtained. (1) The flatness is affected by adding the insulation plate. (2) If distance $d$ between the cathode holder and the bottom plate of the load is long enough, the variance $\eta_{1}$ would be increased when the output voltage is up to a larger threshold value. Contrarily, if $d$ is small enough, the threshold voltage value becomes smaller. (3) The variance $\eta_{2}$ is increased as the distance $d$ is decreasing. (4) If distance $d$ is long enough, the variance $\eta_{3}$ would be decreased when the output voltage is up to a larger threshold value. Conversely, if $d$ is small enough, the threshold voltage value becomes smaller. So, to characterize the IEBA accurately, the distance $d$ could be not too short. 


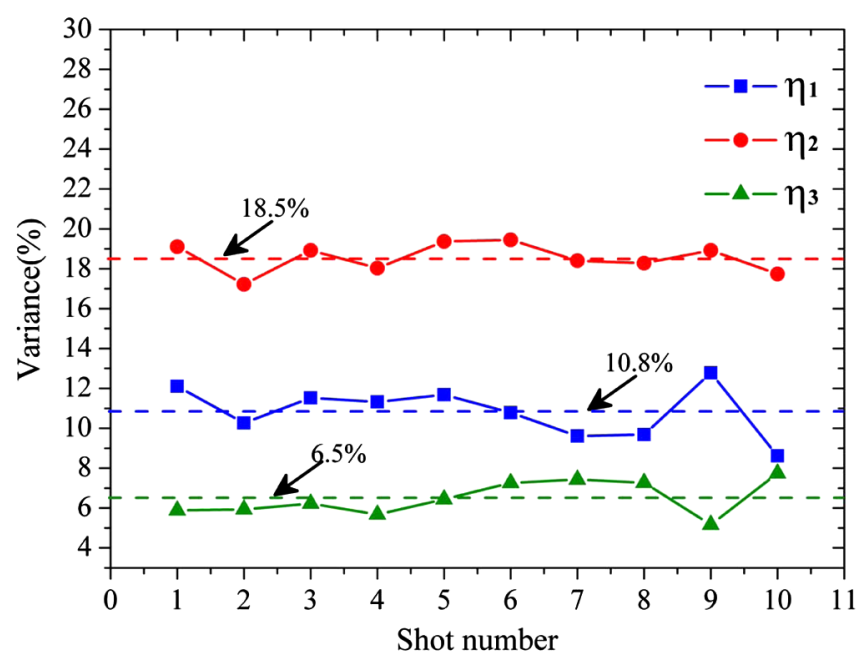

FIG. 10. Variance of the output voltage for ten shots at gas pressure of $0.30 \mathrm{MPa}$ when $6630 \mathrm{DMD}$ polyester film is used.

For these experimental results above, one explanation is that there is field emission somewhere in the vacuum chamber, and the energy is dissipated, so second peaking voltage $\left(V_{2}\right)$ and peaking voltage $\left(V_{P}\right)$ are all decreased. Obviously, the variance $\eta_{3}$ is decreased after adding the insulation plate, and $\eta_{1}$ and $\eta_{2}$ are increased, these are all well in agreement with the experiment results. Indeed, according to analysis of Sec. II', the essence of the difference of the flatness between before and after adding the insulation plate is that the electric field is increased after adding the insulation plate, and the field emission current density of the cathode holder is also increased. So, the voltage is decreased after the first peaking voltage. Of course, the flatness is affected.

To further understand the effect of the insulation plate and to clearly show the field emission of the cathode holder, a piece of 6630 DMD polyester film with thickness of $0.2 \mathrm{~mm}$ consisting of two layers of polyester fiber nonwoven fabric and a single layer of polyester film in the center is pasted on the insulation plate. The same experiment is performed and the gas pressure of the main switch is adjusted to $0.30 \mathrm{MPa}$. After ten shots, the variance for every shot is shown in Fig. 10, and the average variance $\eta_{1}$, $\eta_{2}$, and $\eta_{3}$ of ten shots is about $10.8 \%, 18.5 \%$, and $6.5 \%$, respectively, which is almost the same as that in Figs. 7-9 for 0.30 MPa gas pressure. Also, after ten shots, the surface of the polyester film is changed obviously. Figure 11 shows the photograph of the polyester film before and after experiments.

In Fig. 11, one can see that the insulator paper is ablated after ten shots, and there is a piece of banded mark on the 6630 DMD polyester film, it is just like the electron bombardment of a hollow cathode. It is because the maximum electric field is at the edges of the cathode holder and electrons emit from the edges of the cathode holder. Indeed, the outer layer of the insulator is made of polyester
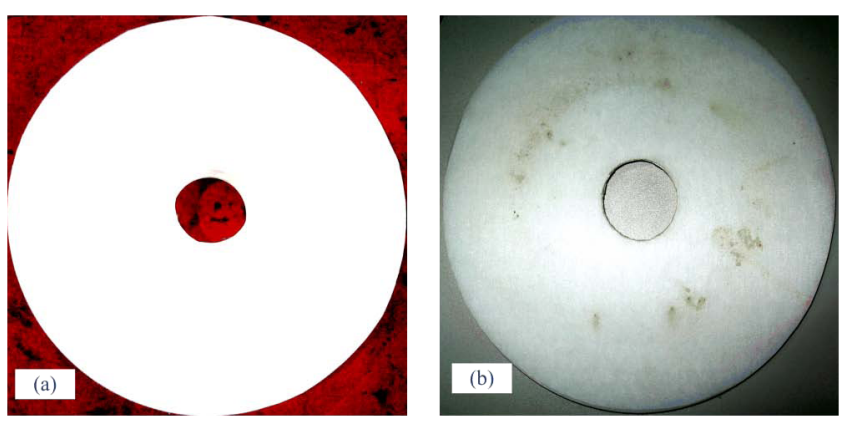

FIG. 11. Photograph of the 6630 DMD polyester film (a) before experiments and (b) after ten shots experiments for $0.30 \mathrm{MPa}$ gas pressure.

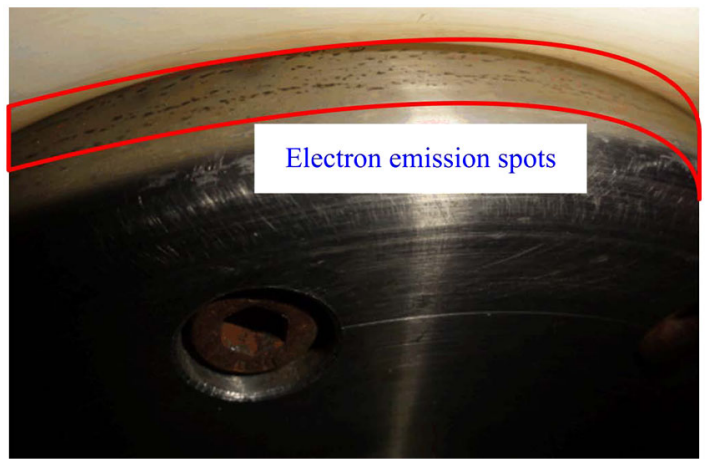

FIG. 12. Photograph of the electron emission spots of the cathode holder.

fiber, and it is very easy to be ablated by the electron bombardment. So after ten shots, there is an obvious mark on the insulator. Also, the electron emission mark on the cathode holder is also observed, which is shown in Fig. 12. There are many spots at the edge of the cathode holder. These all prove that there exists field emission on the cathode holder, which is one of the important factors affecting the flatness of the output voltage on the load.

\section{CONCLUSION}

In summary, the electron emission phenomenon of the cathode holder of a vacuum diode under hundreds of kilovolts for a pulsed-power modulator was investigated by using an axial current water load, and its effect on the flatness of the output voltage was investigated by experiment before and after adding an insulation plate when a water load is used. It was found that: (1) the flatness was affected by adding the insulation plate. (2) If distance $d$ between the cathode holder and the bottom plate of the load was long enough, the variance $\eta_{1}$ would be increased when the output voltage was up to a larger threshold value. Contrarily, if $d$ was small enough, the threshold voltage value could become smaller. (3) The variance $\eta_{2}$ was increased as the distance $d$ decreasing. (4) If distance $d$ 
was long enough, the variance $\eta_{3}$ would be decreased when the output voltage was up to a larger threshold value. Conversely, if $d$ was small enough, the threshold voltage value becomes smaller. So, to characterize the IEBA accurately, the distance $d$ could not be too short.

Further, according to the analysis and electric field calculation, it was found that the field emission at the edges of the cathode holder was the main reason to cause the change of the flatness of the output voltage. In other words, after adding the insulation plate, the electric field at the cathode holder was increased, so electron emission was enhanced and field emission current density was increased. Of course, the flatness of the output voltage was also changed. At last, a piece of DMD polyester film is used to further show the field emission of the cathode holder, and a photograph of DMD polyester film by electron bombardment clearly proves that the electrons were emitted from the edges of the cathode holder. So to improve the quality of the output voltage, the electron emission of the cathode holder should be avoided.

\section{ACKNOWLEDGMENTS}

The authors gratefully acknowledge the support from the Fund of Innovation, Graduate School of NUDT under Grant No. B090701. This work is also supported by Hunan Provincial Innovation Foundation for Postgraduate.

[1] S. H. Gold and G. S. Nusinovich, Rev. Sci. Instrum. 68, 3945 (1997).

[2] Y. J. Chen, A. A. Neuber, J. Mankowski, J. C. Dickens, M. Kristiansen, and R. Gale, Rev. Sci. Instrum. 76, 104703 (2005).

[3] Y. W. Fan, H. H. Zhong, H. W. Yang, Z. Q. Li, T. Shu, J. Zhang, Y. Wang, and L. Luo, J. Appl. Phys. 103, 123301 (2008).

[4] R. Menon, A. Roy, S. K. Singh, S. Mitra, V. Sharma, S. Kumar, A. Sharma, K. V. Nagesh, K. C. Mittal, and D.P. Chakravarthy, J. Appl. Phys. 107, 093301 (2010).

[5] R. C. Hazelton, E. J. Yadlowsky, J. J. Moschella, E. P. Carlson, C. Vidoli, J. Niemel, B.H. Failor, P. L.
Coleman, J.S. Levine, Y. Song, H. M. Sze, and J. W. Thornhill, IEEE Trans. Plasma Sci. 31, 1355 (2003).

[6] S. V. Lebedev, F. N. Beg, S. N. Bland, J.P. Chittenden, A. E. Dangor, M. G. Haines, K. H. Kwek, S. A. Pikuz, and T. A. Shelkovenko, Phys. Plasmas 8, 3734 (2001).

[7] T. A. Shelkovenko, D. A. Chalenski, K. M. Chandler, J. D. Douglass, J. B. Greenly, D. A. Hammer, B. R. Kusse, R. D. McBride, and S. A. Pikuz, Rev. Sci. Instrum. 77, 10F521 (2006).

[8] W. A. Stygar, M. E. Cuneo, D. I. Headley, H. C. Ives, R. J. Leeper, M. G. Mazarakis, C. L. Olson, J. L. Porter, T. C. Wagoner, and J.R. Woodworth, Phys. Rev. ST Accel. Beams 10, 030401 (2007).

[9] J.D. Sethian, M. Myers, I. D. Smith, V. Carboni, J. Kishi, D. Morton, J. Pearce, B. Bowen, L. Schlitt, O. Barr, and W. Webster, IEEE Trans. Plasma Sci. 28, 1333 (2000).

[10] J. P. Apruzese, J. L. Giuliani, M. F. Wolford, J. D. Sethian, G. M. Petrov, D. D. Hinshelwood, M. C. Myers, and D. M. Ponce, Appl. Phys. Lett. 88, 121120 (2006).

[11] H. Ito, K. Lgawa, I. Kitamura, and K. Masugata, Rev. Sci. Instrum. 78, 013502 (2007).

[12] J. Benford, J. Swegle, and E. Schamiloglu, High Power Microwaves (Taylor \& Francis, New York, 2007), 2nd ed.

[13] F. Hegeler, J. L. Giuliani, Jr., J. D. Sethian, M. C. Myers, M. F. Wolford, P. M. Burns, and M. Friedman, IEEE Trans. Plasma Sci. 36, 778 (2008).

[14] X. B. Cheng, J.L. Liu, H. B. Zhang, J. H. Feng, and B. L. Qian, Phys. Rev. ST Accel. Beams 13, 070402 (2010).

[15] J. Han, J. H. Kim, S. D. Park, M. Yoon, and S. Y. Park, Phys. Rev. ST Accel. Beams 12, 113501 (2009).

[16] Y. Yin, H. H. Zhong, J. L. Liu, H. M. Ren, J. H. Yang, X. P. Zhang, and Z. Q. Hong, Rev. Sci. Instrum. 81, 093301 (2010).

[17] J. L. Liu, Y. Yin, B. Ge, T. W. Zhan, X. B. Chen, J. H. Feng, T. Shu, J.D. Zhang, and X.X. Wang, Laser Part. Beams 25, 593 (2007), http://journals.cambridge.org/ abstract_S0263034607000705.

[18] R. H. Fowler and L. W. Nordheim, Proc. R. Soc. A 119, 173 (1928).

[19] B. M. Cox and W. T. Williams, J. Phys. D 10, L5 (1977). 